\title{
Femtosecond-Laser-Written S-Curved Waveguide in Nd:YAP Crystal: Fabrication and Multi-Gigahertz Lasing
}

\author{
Lingqi Li ${ }^{\circledR}$, Ziqi Li ${ }^{\circledR}$, Weijie Nie, Carolina Romero ${ }^{\circledR}$, Javier R. Vázquez de Aldana ${ }^{\circledR}$, \\ and Feng Chen ${ }^{\circledR}$, Senior Member, OSA
}

\begin{abstract}
We report on the design and fabrication of straight and S-curved waveguides in neodymium-doped yttrium aluminate (Nd:YAP) crystal by using direct femtosecond laser writing. These S-curved channel waveguides are based on the hexagonal optical-lattice-like and depressed cladding geometry. For each type waveguide, S-curves with three lateral offsets of $50 \mu \mathrm{m}, 100 \mu \mathrm{m}$, and $150 \mu \mathrm{m}$ are implemented. These waveguides are with good guiding properties and low bending losses. With $\mathrm{S}$-curved waveguide as laser cavity, dual-wavelength, $31.6 \mathrm{GHz}$ waveguide laser operating at $1064 \mathrm{~nm}$ and $1079 \mathrm{~nm}$ have been demonstrated based on $\mathrm{MoS}_{2}$ as a saturable absorber. This work paves the way to develop new on-chip ultrafast laser sources based on femtosecond laser inscribed S-curved waveguides.
\end{abstract}

Index Terms-Curved waveguide, femtosecond laser writing, mode-locked lasers, optical waveguide.

\section{INTRODUCTION}

W AVEGUIDE-BASED monolithic laser source could be on-chip integrated within photonics devices with miniature and compact cavity, opening the new possibilities for integrated optics. [1]-[5]. Micron-scale waveguide structures could confine light propagation in very small volumes, which, in turn, results in high optical intra-cavity intensities compared to original bulk material. Consequently, superior laser performances, such as lower lasing threshold and enhanced slope efficiency could be expected in various waveguide structures [6]-[8]. Nevertheless, most waveguide configuration focus on the straight channels, with the drawback of limiting the actual spatial control

Manuscript received May 10, 2020; revised July 25, 2020; accepted August 7, 2020. Date of publication August 12, 2020; date of current version December 15, 2020. This work was supported in part by the National Natural Science Foundation of China under Grant 61775120 and Grant MINECO FIS201787970-R, in part by the Major Program of Shandong Province Natural Science Foundation under Grant ZR2018ZB0649, and in part by the Ministerio de Economía y Competitividad de España under Grant MAT2016-75362-C3-1-R. (Corresponding author: Feng Chen.)

Lingqi $\mathrm{Li}$, Ziqi $\mathrm{Li}$, Weijie Nie, and Feng Chen are with the School of Physics, and State Key Laboratory of Crystal Materials, Shandong University, Jinan 250100, China (e-mail: lingqilee@163.com; drziqili@163.com; weijienie@163.com; drfchen@sdu.edu.cn).

Carolina Romero and Javier R. Vázquez de Aldana are with the Aplicaciones del Láser y Fotónica Group, Departamento de Física, Universidad de Salamanca, 37008 Salamanca, Spain (e-mail: carolbaiona@gmail.com; jrval@usal.es).

Color versions of one or more of the figures in this article are available online at https://ieeexplore.ieee.org.

Digital Object Identifier 10.1109/JLT.2020.3015690 of the light transmission. In many applications of compact integrated optic networks, such as wavelength multiplexers, directional couples, analogto-digital converters, high-gain amplifiers, optical switches etc, curved waveguide is necessary to connect two different directions straight waveguide that are offset with each other and introduce a lateral separation between adjacent ports [9]-[11]. Among them, S-curved channel waveguide with advantage of low transition loss and easier to implement, are particularly desirable for the construction of complicated monolithic photonic devices like lossless beam splitters or ring lasers in crystalline materials [12], [13]. Therefore, the fabrication of smooth S-bend shaped waveguides with low bending loss is in desired.

Recently, femtosecond laser writing is considered as a powerful technique to fabricate versatile 3D waveguide configurations towards diverse applications, showing the superiority of wide applicability of materials, negligible effect of thermal-diffusion, and capability for maskless 3D processing [14]-[19]. During the processing of femtosecond laser writing, permanent and stable refractive-index modification may be created in the focal volume through nonlinear processes such as tuning ionization, multiphoton absorption and avalanche ionization [20], [21], which could be used for waveguide fabrication. There are two different refractive-index change regimes. Type I modification is induced with positive refractive index change $(\Delta n>0)$, in which the waveguide core is just located in the laser irradiated region. In these waveguides, the luminescence and nonlinear properties of original bulk material is disrupted, limiting some lasing and nonlinear application [22], [23]. By contrast, Type II modification with negative refractive change $(\Delta n<0)$ permits waveguide core situated in the surrounding region of laser-written tracks. In addition, this modification allows one to arbitrarily design waveguide geometry using these low-index laser-written tracks. Typically, depressed cladding waveguide geometries, encircled by several reduced refractive index tracks, are favorable configuration with relatively low loss and ease to integrate with optical fibers [24]. Furthermore, photonic-lattice-like waveguide is performed with undamaged waveguide core surrounded by many laser-written track layers, capable of mode tailoring [25].

The neodymium-doped yttrium aluminate $\left(\mathrm{Nd}: \mathrm{YAlO}_{3}\right.$ or Nd:YAP) crystal exhibits distinguished optomechanical properties, which makes it an ideal candidate material for single cavity dual-wavelength lasing [26]-[29]. Recently, multi-gigahertz 


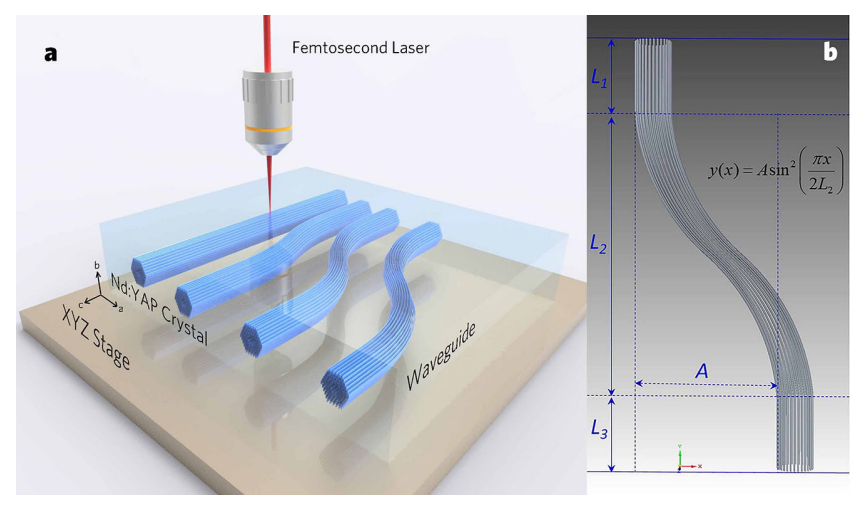

Fig. 1. (a) Schematic plot of the S-curved hybrid optical-lattice-like waveguides fabrication process with the femtosecond laser direct writing. (b) Schematic of the designed curved waveguides. The " $A$ " parameter is the "separation" indicated in the samples report (50 $\mu \mathrm{m}, 100 \mu \mathrm{m}$ and $150 \mu \mathrm{m})$.

mode-locked lasers have attracted increasing research attention due to a large variety of applications, ranging from precision metrology, ultrafast nonlinear spectroscopy, to high-speed optical communication [30]-[32]. Molybdenum disulfide $\left(\mathrm{MoS}_{2}\right)$ is one of the well-developed 2D materials that can be used as excellent broadband saturable absorber [33]-[35]. Recently, continuous-wave (CW) or Q-switched mode-locked (QML) waveguide lasers operated have been demonstrated by many groups with repetition rate of $1.5 \mathrm{GHz}$ [36], $5.9 \mathrm{GHz}$ [37], $6.5 \mathrm{GHz}$ [38], $7.8 \mathrm{GHz}[39], 8.8 \mathrm{GHz}$ [40] and $21 \mathrm{GHz}$ [41] at different gain medium, such as Nd:YAG, $\mathrm{Nd}: \mathrm{YVO}_{4}, \mathrm{Yb}: \mathrm{YAG}$, $\mathrm{Ho}^{3+}:$ YAG, and Ti:sapphire. The CW dual-wavelength Nd:YAP waveguide laser has been realized [42], whilst in pulsed regime such waveguide laser has not been implemented.

In this work, we present the fabrication and characterization of straight and S-curved channel waveguides in Nd:YAP crystal by femtosecond direct writing. These waveguides possess the cross-sectional of cladding and hexagonal optical-lattice-like geometry. We design three types S-curved waveguide with different lateral offset of 50,100 and $150 \mu \mathrm{m}$. Using the S-curved waveguide as laser cavities, we realize $31.6-\mathrm{GHz}$ waveguide lasers at dual-wavelength of $1064 \mathrm{~nm}$ and $1079 \mathrm{~nm}$ with duration as short as $16 \mathrm{ps}$ and the signal-to-noise up to $49 \mathrm{~dB}$, which exhibits the high pulsed laser performances in the femtosecond laser-written waveguide platforms.

\section{EXPERIMENT IN DETAILS}

The Nd:YAP crystal used in this work was cut into the dimensions of $2 \mathrm{~mm} \times 8 \mathrm{~mm} \times 10 \mathrm{~mm}(b \times c \times a)$. Fig. 1(a) depicts the fabrication process of straight and S-curved waveguides by using femtosecond laser writing. We used a Ti:Sapphire amplified laser system (Spitfire, Spectra Physics), in which delivered $120 \mathrm{fs}$ duration pulses, $796 \mathrm{~nm}$ central wavelength, and with $1 \mathrm{kHz}$ repetition rate, to fabricate the buried waveguide from the largest surface $(10 \mathrm{~mm} \times 10 \mathrm{~mm})$. A calibrated neutral density filter and a half-wave plate was used to control the irradiate pulse energy. Several tests at different pulse energies and scanning speed was performed to find the optimum fabrication parameters. The femtosecond laser beam was focused by a $20 \times$ microscope objective $(\mathrm{NA}=0.40)$. The sample was placed at a PC-controlled 3D motorized stage with a spatial resolution of $100 \mathrm{~nm}$ and was scanned at a constant velocity along $c$-axis orientation. This processing allows a low refractive-index track formation inside the sample, which could be used to construct waveguides. For hexagonal optical-lattice-like waveguides, a pulse energy of $0.48 \mu \mathrm{J}$ was selected to fabricate waveguides with a scanning speed of $500 \mu \mathrm{m} / \mathrm{s}$. After that, four-layer laser-written low-index boundaries were performed to construct the hybrid optical-lattice -like geometry with a number of parallel tracks (10 $\mu$ m lateral separation of adjacent parallel tracks), which were written at different positions of sample. We have also fabricated depressed cladding waveguides with a pulse energy of $0.8 \mu \mathrm{J}$ at a scan speed of $500 \mu \mathrm{m} / \mathrm{s}$. Several tracks were written at different depth of sample with $3 \mu$ m lateral separation to produce the desired circular geometry of the cladding tracks. Fig. 1(b) illustrates the schematic of the curved waveguides. As one can see, the S-curved waveguide consists of two straight part and one $\mathrm{S}$-curved part. The parameter $A$ is the lateral offset between the input and output ports. In the case of the YAP samples, $L_{1}$ $=1 \mathrm{~mm}, L_{2}=8 \mathrm{~mm}, L_{3}=1 \mathrm{~mm}$. For the smooth S-curved waveguide channel, in order to optimize the bend loss and radiation loss caused by curvature radius changes of S-shape curve, the waveguides design is based on sine-cosine function. We have also implemented the S-curve with two consecutive circular-arcs and a reduction of about $10 \%$ was obtained in the losses. In our work, the fabrication of S-curved waveguides was parameterized with a $\sin ^{2} \theta$ function:

$$
y(x)=A \sin ^{2}\left(\frac{\pi x}{2 L_{2}}\right)
$$

Where $L_{2}$ is the length of the S-curved part. The 3D motorized stage movement of the $x$-axis and $y$-axis follows this function. As the $x$-axis moves laterally, the $y$-axis would produce offset along the vertical axis. It is particularly useful for the extension of our devices to 3D structures or more complex elements such as Mach-Zehnder interferometers. With the hexagonal photonic lattice and circular cladding cross-section configuration, both straight and S-curved waveguides with three type lateral offset of 50,100 , and $150 \mu \mathrm{m}$ were successfully fabricated with same writing parameters.

The Q-switched mode-locked waveguide laser operation in this work was performed by utilizing a typical end-face coupling configuration. A tunable CW Ti:sapphire laser (Coherent MBRPE) was utilized as the linearly polarized pump source at $813 \mathrm{~nm}$. The pump polarization was controlled by a half-wave plate. In order to couple the pump beam into the microscale cladding waveguide efficiently, the pump beam was converged to the input facet of sample by a spherical convex lens with a focal length of $25 \mathrm{~mm}$. For the implementation of Q-switched mode-locking, an input mirror $\mathrm{M}_{1}$ with high transmission (98\%) at $813 \mathrm{~nm}$ and high reflectivity (99\%) at $1064 \mathrm{~nm}$, and an output mirror $\left(\mathrm{M}_{2}\right)$ with high reflectivity of $99 \%$ at $813 \mathrm{~nm}$ and high transmission of $90 \%$ at $1064 \mathrm{~nm}$, constructed the Fabry-Perot resonant cavity. The $\mathrm{MoS}_{2}$ thin film is a commercial product (2D materials 
supplier (6Carbon Technology, Shenzhen, China), which was directly attached to the output facet of the waveguides. The generated laser was collected with an objective lens (N.A. = 0.4 ) and a long pass filter (Thorlabs, FEL0850) of $850 \mathrm{~nm}$ cut-off wavelength was utilized to eliminate the un-absorbed pump laser. The QML laser was then coupled into a single mode fiber, which was directly connected to a high-speed InGaAs photodetector (New focus, 1414 model) and real-time digital oscilloscope (Tektronix, MSO 72504DX). The model profile was imaged by an infrared CCD, and the emission spectra of waveguide laser was analyzed by a spectrometer (Zolix, SGM100 Spectrograph) with resolution of $0.2 \mathrm{~nm}$.

In order to experimentally characterize the properties of these S-curved waveguides, an end-face coupling set up is exploited to determine the modal profiles and propagation loss. The $\mathrm{CW}$ 1064-nm solid-state laser was coupled into and out of the endface of sample by a pair $20 \times$ microscope object lens. Then, the model profile of the output transmitted light was collected and imaged by a CCD camera and analyzed by the RayCi software.

An optical microscope (Olympus BX43) integrated with a 532 nm-laser-beam was employed as excitation source to investigate the fluorescence properties. The Nd: YAP sample was placed on a precision XYZ motorized stages $(0.1-\mu \mathrm{m}-$ resolution). The laser beam was focused onto the polished cross section of sample with microscope objective lens $(100 \times, 0.8$ NA). The back-scattered emission signal of $\mathrm{Nd}^{3+}$ ions was obtained with the same objective lens. After passing through a filter (cut-off at $186 \mathrm{~cm}^{-1}, \mathrm{OD}>6 @ 532 \mathrm{~nm}$ ), lenses and pinholes, the signal was coupled into a $100 \mu \mathrm{m}$ core fiber and analyzed via a high-resolution spectrometer $\left(<8 \mathrm{~cm}^{-1}\right)$.

\section{RESUlTS AND DiscUSSION}

\section{A. Characterization of the Waveguides}

Fig. 2(a) shows the top view schematic diagram of straight and S-curved waveguides. In this work, we design and fabricate four types waveguides of straight and S-curved with lateral offset of $50\left(A_{1}\right), 100\left(A_{2}\right)$, and $150 \mu \mathrm{m}\left(A_{3}\right)$. Using an optical microscope (Axio Imager, Carl Zeiss), the cross-sectional microscope images of hexagonal optical-lattice-like waveguides Nos. 1 to 4 and cladding waveguides Nos. 5 to 10, corresponding to straight and S-curved waveguides, respectively, which is exhibited in Fig. 2(b) and 2(c). The waveguide core of hexagonal opticallattice-like waveguides is located at the undamaged region surrounded by four layer laser-written tracks with area of $30 \times 30$ $\mu \mathrm{m}^{2}$, as identified by the red dish line in Fig. 2(b). The diameter of all the cladding waveguides are $100 \mu \mathrm{m}$.

The resulting near-field mode distributions of photonic lattice waveguides and cladding waveguides are depicted in Fig. 3 and Fig. 4, respectively. As one can see, the waveguide Nos. 1-3 shows better guiding confinement along both TE and TM polarization. Whereas, the light propagation of the waveguide No. 4 with $150 \mu \mathrm{m}$ lateral offset is not well confined. By contrast, the cladding waveguides show better guiding confinement than optical-lattice-like ones even at large lateral offsets of $100 \mu \mathrm{m}$ and $150 \mu \mathrm{m}$. As one can see, the cladding waveguides show multi-mode distributions, which can be well understood due to

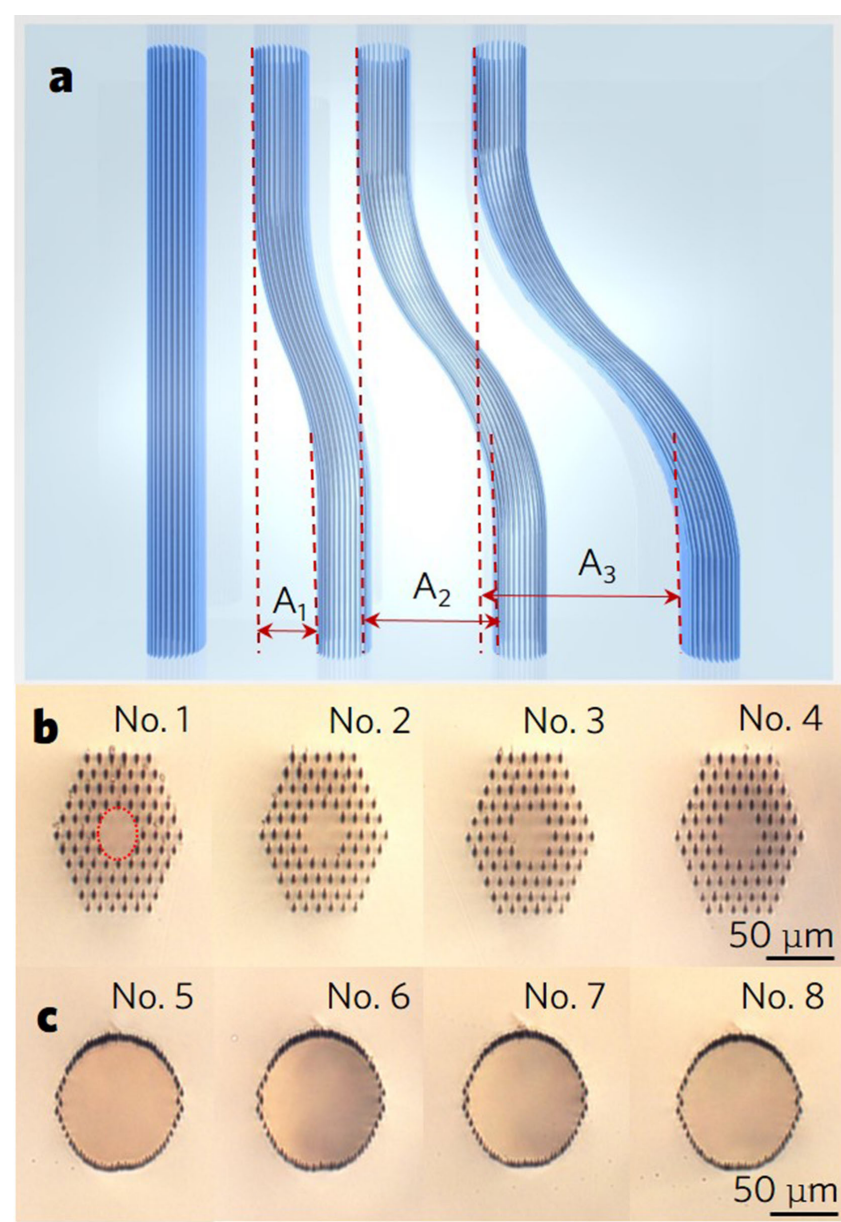

Fig. 2. (a) Top view schematic plot of the straight and S-curved waveguides with different lateral offset of $A_{1}(50 \mu \mathrm{m}), A_{2}(100 \mu \mathrm{m})$ and $A_{3}(150 \mu \mathrm{m})$, respectively. (b) and (c) Optical microscope cross-sectional image of hybrid optical-lattice-like waveguides and cladding waveguides No. 1 and No. 5 (straight), No. 2 and No. $6\left(A_{1}=50 \mu \mathrm{m}\right)$, No. 3 and No. $7\left(A_{2}=100 \mu \mathrm{m}\right)$, and No. 4 and No. $8\left(A_{3}=150 \mu \mathrm{m}\right)$, respectively.
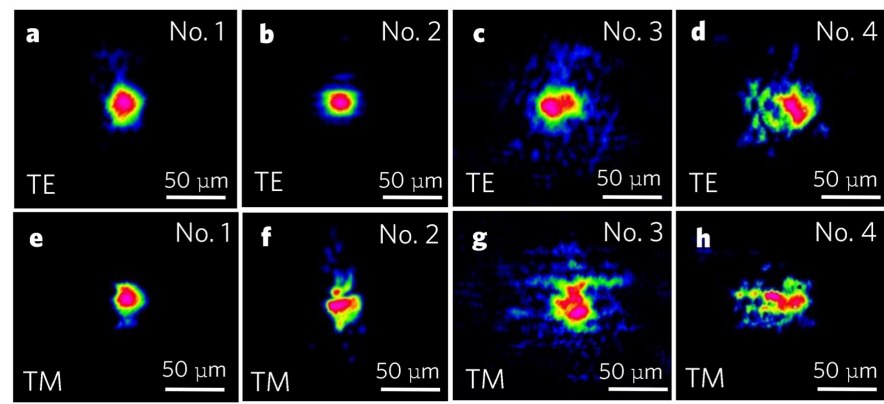

Fig. 3. (a) Measured near-field modal profiles of hybrid optical-lattice-like waveguides at $1064 \mathrm{~nm}$. (a) and (e) No. 1, (b) and (f) No. 2, (c) and (g) No. 3, (d) and (h) No. 4, along TE (top) and TM (bottom) polarization, respectively.

the large diameter of the cladding structures in comparison to the test wavelength.

As one of the most important factors for waveguide transmission, we have investigated the propagation loss of optical-latticelike waveguides and cladding waveguides at wavelength of 1.064 


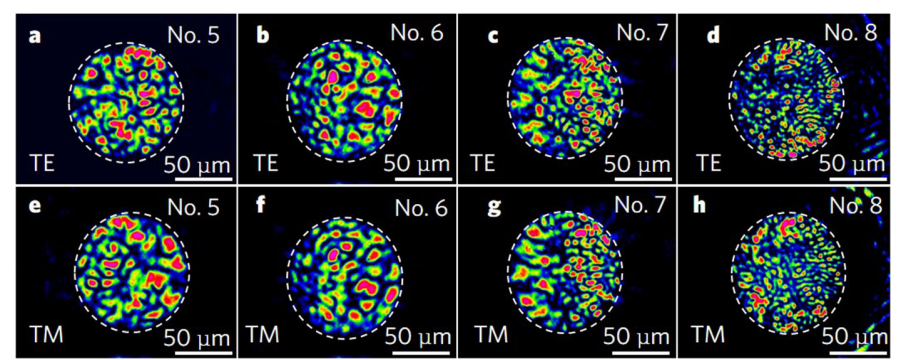

Fig. 4. (a) Measured near-field modal profiles of cladding waveguides at $1064 \mathrm{~nm}$. (a) and (e) No. 5, (b) and (f) No. 6, (c) and (g) No. 7, (d) and (h) No. 8, along TE (top) and TM (bottom) polarization, respectively. The dotted circles are the sign of the laser-written tracks.

TABLE I

Propagation Loss of Straight AND S-CURVEd WAVEguideS

\begin{tabular}{cccc}
\hline \hline & Waveguide & TM $(\mathrm{dB} / \mathrm{cm})$ & $\mathrm{TE}(\mathrm{dB} / \mathrm{cm})$ \\
\hline & No. 1 & 1.12 & 1.11 \\
Optical-lattice-like & No. 2 & 1.43 & 1.44 \\
waveguide & No. 3 & 1.67 & 1.63 \\
& No. 4 & 2.13 & 2.12 \\
\hline & No. 5 & 0.67 & 0.64 \\
Cladding waveguide & No. 6 & 0.85 & 0.83 \\
& No. 7 & 1.04 & 0.99 \\
& No. 8 & 1.23 & 1.12 \\
\hline \hline
\end{tabular}

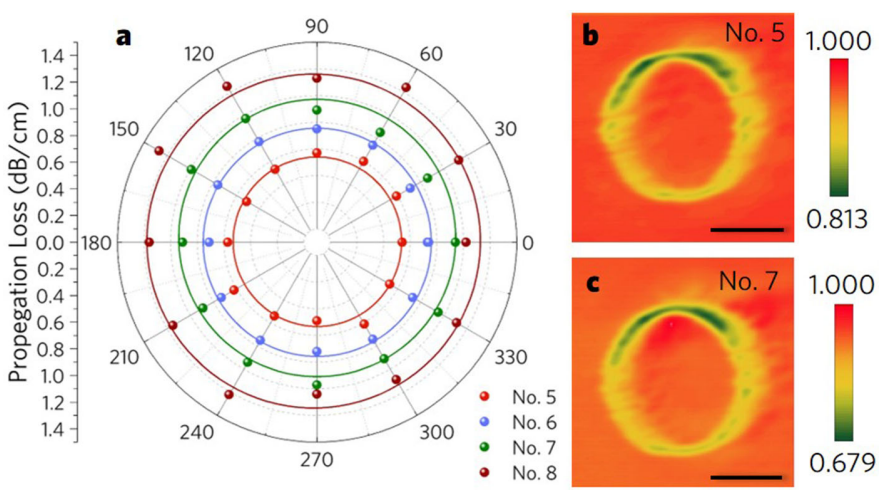

Fig. 5. (a) Polarization images of the propagation loss of cladding waveguides No. 5 to No. 8 at $1064 \mathrm{~nm}$. (b) and (c) Micro-photoluminescence measurements. The spatial distributions of emitted intensity obtained from the end face of straight waveguide No. 5 (b) and S-curved waveguide No.7 (c).

$\mu \mathrm{m}$, which is exhibited in Table I. It is clearly that the propagation loss of cladding waveguides is smaller than photonic lattice waveguide. The possible reason for the lower propagation loss of cladding waveguide is the large scale of structure and the closer arranged laser-inscribed tracks, which can lead to more tight confinement on light propagation. In order to thoroughly investigate the polarization properties of the waveguides, all-angle light transmission of guidance has been depicted in Fig. 5(a). As one can see, the cladding waveguides support light transmission at any polarizations, showing good properties of polarization-insensitive. Optical-lattice-like waveguides also exhibit the polarization-insensitive features in our experiment. The bend loss of S-curved waveguide is obtained by comparing the propagation loss of straight and S-curved waveguides written via the same laser parameters. For the cladding waveguides, the bend loss of waveguides Nos. 6 to 8 is $0.18,0.37$ and $0.56 \mathrm{~dB}$ of the lateral offset of 50,100 and $150 \mu \mathrm{m}$ at TM polarization, respectively. The value at TE polarization is $0.19,0.35$ and $0.48 \mathrm{~dB}$. As for hexagonal optical-lattice-like waveguides, the bend loss of Nos. 2 to 4 is $0.31 \mathrm{~dB}(0.33 \mathrm{~dB}), 0.55 \mathrm{~dB}(0.52 \mathrm{~dB})$ and $1.01 \mathrm{~dB}(1.01 \mathrm{~dB})$ at TM (TE) polarization, respectively. The largest bend loss of waveguide No. 8 with $150 \mu \mathrm{m}$ lateral offset is less than $\sim 0.6 \mathrm{~dB}$, which indicates the possibility of application in compact on-chip photonics. Figs. 5(b) and 5(c) depict the fluorescence intensity maps of ${ }^{4} \mathrm{~F}_{3 / 2} \rightarrow{ }^{4} \mathrm{~F}_{9 / 2}$ emission line at $\sim 893 \mathrm{~nm}$ of cladding waveguide No. 5 and No. 7. As one can see, the original properties of Nd:YAP crystal in the waveguide core has not been damaged in the process of femtosecond laser writing. But in the region of laser-written tracks, the fluorescence intensity is decreased. Similar fluorescence properties are obtained from the optical-lattice-like waveguides. By comparing the fluorescence intensity of straight waveguide No. 5 and S-curved waveguide No. 7, we can see that femtosecond laser-induced damage creation is more relevant in S-curved waveguide No. 7 than in straight waveguide No. 5 .

\section{B. Q-Switched Mode-Locked Dual-Wavelength Waveguide Laser at S-Curved Waveguide}

Using the straight and S-curved waveguides as laser cavities, the dual-wavelength mode-locked laser could operate in the Nd:YAP crystal with $\mathrm{MoS}_{2}$ as the saturable absorber, which is shown in the schematic diagram Fig. 6(a). Fig. 6(b) shows the measured average output power as a function of pump power of cladding waveguides No. 5 (straight) and No. 7 (S-curved). The slope efficiency implies the conversion efficiency of the gain medium to convert the energy of the pump into output lasers, defined as the slope of the line obtained by plotting the laser output versus the pump power. As one can see from the linear fit, the laser oscillation threshold of straight and S-curved waveguides is $132 \mathrm{~mW}$ and $196 \mathrm{~mW}$ with the slope efficiencies of $24.3 \%$ and $14.1 \%$, reaching the maximum output power of $150 \mathrm{~mW}$ and $77 \mathrm{~mW}$, respectively. The standard deviation of straight waveguide fitted data is 8.3 , and the standard deviation of S-curved waveguide fitted data is 4.6. The slope efficiency of the S-curved cladding waveguide laser is $\sim 58 \%$ of the value for the straight waveguide, which is due to the additional bending loss and the curved laser cavity effect for S-curved waveguide. Fundamental QML has been obtained once the pump power exceeds the laser oscillation threshold. Efficient harmonic mode-locking up to 4th of the fundamental repetition frequency waveguide laser emission has been achieved in the straight waveguide when the pump power higher than $229 \mathrm{~mW}$ ( $\sim 263 \mathrm{~mW}$ at S-curved waveguide). The excited laser spectra of $1064 \mathrm{~nm}$ and $1079 \mathrm{~nm}$ in S-curved waveguide No. 7 are shown in Fig. 7, which corresponds to the main emission lines of ${ }^{4} \mathrm{~F}_{3 / 2} \rightarrow{ }^{4} \mathrm{~F}_{11 / 2}$ transition correlated to $\mathrm{Nd}^{3+}$ ions. The insets in Fig. 7 depict the mode profiles of the generated laser. The modal profiles of the generated lasers are not uniform, which is 

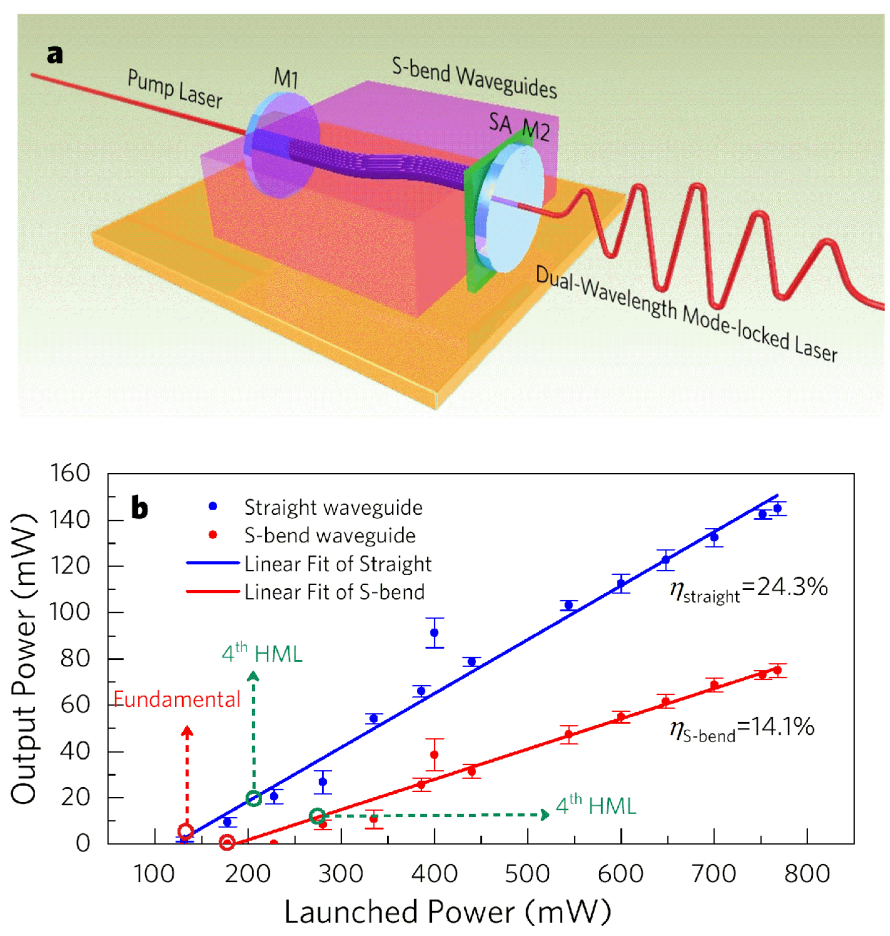

Fig. 6. (a) Prototype and spatial configuration of the monolithic Q-switched mode-locked waveguide laser. (b) The average output power as a function of the input power of cladding waveguides No. 5 (straight) and No. 7 (S-curved).
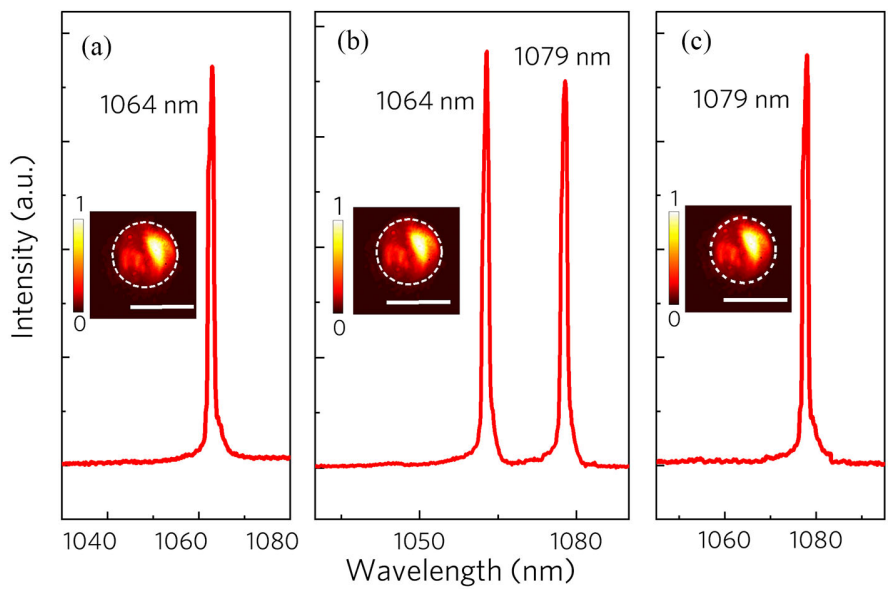

Fig. 7. Spectra of QML laser emissions (a) at single wavelength of $1064 \mathrm{~nm}$, (b) the simultaneous dual-wavelength of 1064 and $1079 \mathrm{~nm}$, and (c) the single wavelength of $1079 \mathrm{~nm}$. The inset is the measured near-field modal profile of the output laser. The scale bars in the bottom right of all images are $100 \mu \mathrm{m}$.

due to the inhomogeneity of the laser-induced refractive index modification in the cladding waveguide.

By changing the polarization of pump beam, one can achieve both single- (1064 or $1079 \mathrm{~nm})$ and dual-wavelength (1064 and $1079 \mathrm{~nm}$ ) lasers. When the pumping laser is at TE polarization (corresponding to the $0^{\circ}$ ), the single-wavelength laser at $1079 \mathrm{~nm}$ is observed, as shown in Fig. 7(a). When the polarization is
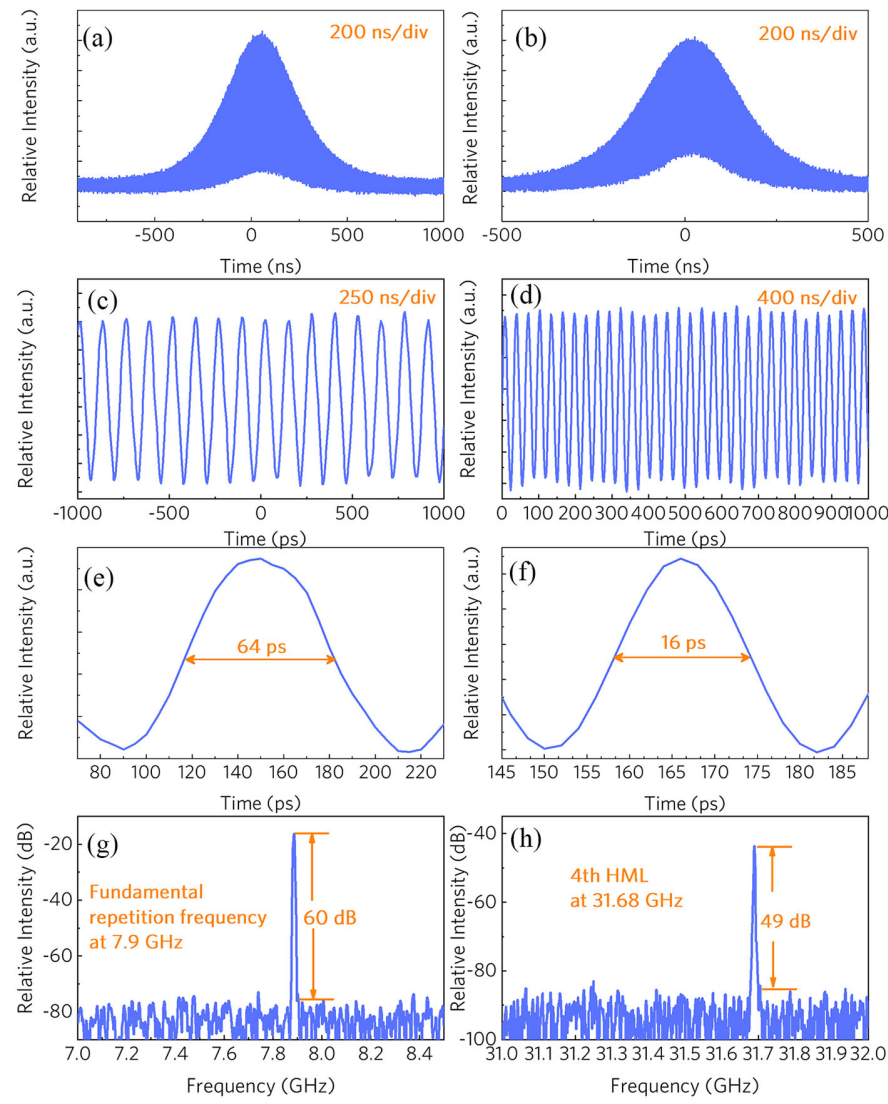

Fig. 8. Characterization of QML of S-curved cladding waveguide No. 7 at 1064 and $1079 \mathrm{~nm}$. Fundamental mode-locked laser performance (a) Q-switched envelope on a nanosecond timescale. (c) Mode-locked pulse trains on picosecond timescale. (e) Single pulse train of 64 ps, and (g) measured RF spectrum. (b), (d), (f) and (h) correspond to fourth harmonic performance.

changed to $45^{\circ}$, the simultaneous dual-wavelength laser generation is obtained with an intensity ration of $\sim 1: 1$ (see Fig. 7(b)). When the pump light is at TM polarization, i.e., corresponding to $90^{\circ}$ in Fig. 7(c), the single-wavelength laser operation at $1064 \mathrm{~nm}$ is achieved. This tuning method is suitable for both cladding and hexagonal optical-lattice-like waveguides. And for the dual-wavelength laser, the power ratio of 1064 and $1079 \mathrm{~nm}$ is tunable, which is in good agreement with the Nd:YAP CW waveguide laser [42].

Fig. 8 shows the typical mode-locked operation of S-curved cladding waveguide No. 7 at dual-wavelength at 1064 and $1079 \mathrm{~nm}$. Both fundamental repetition of $7.9 \mathrm{GHz}$ (left part of Fig. 8) and harmonic QML up to 4th of the fundamental repetition frequency (right part of Fig. 8) waveguides lasers are generated under the pump power of $183 \mathrm{~mW}$ and $264 \mathrm{~mW}$, respectively. The single Q-switched envelope with mode-locked trains on a nanosecond scale of $200 \mathrm{~ns} / \mathrm{div}$ has been demonstrated in Fig. 8(a) and 8(b). Figs. 8(c) and 8(d) present the mode-locked trains on nanosecond scale. Single mode-locked trace is shown in Fig. 8(e) with full width at half-maximum (FWHM) as short as $64 \mathrm{ps}$. The pulse duration of fourth harmonic performance is $16 \mathrm{ps}$, corresponding to a quarter as short as fundamental 

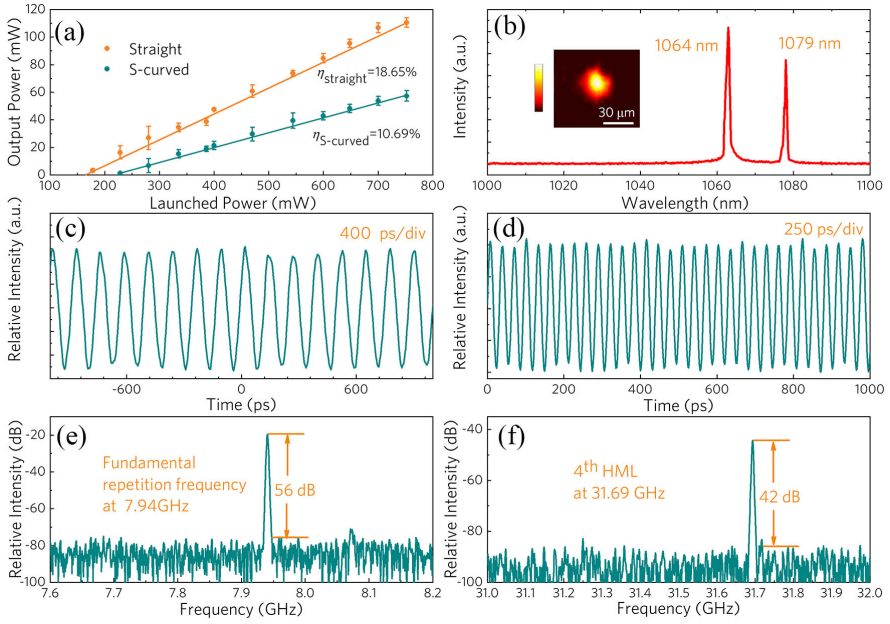

Fig. 9. Mode-locked performance of S-curved hexagonal optical-lattice-like waveguide No. 3. (a) The average output power as a function of the input power (b) Spectra of QML laser emissions at dual-wavelength $1064 \mathrm{~nm}$ and $1079 \mathrm{~nm}$. Inset is the modal profile of the output laser. (c) Fundamental QML pulse trains recorded in $400 \mathrm{ps} / \mathrm{div}$ and (e) the RF spectrum. (d) and (f) correspond to fourth harmonic QML.

operation, which is illustrated in Fig. 8(f). The measurement of radio frequency $(\mathrm{RF})$ spectrum of the output laser is carried out using a Fourier transform of the signal obtained on an oscilloscope. Fundamental performance is operated at $7.90 \mathrm{GHz}$ with the signal-to-noise (SNR) of $60 \mathrm{~dB}$, which is shown in Fig. 8(g). As we can see from Fig. 8(h), the repetition rate has been up to $31.68 \mathrm{GHz}$, corresponding to the 4 th harmonic of the $7.9 \mathrm{GHz}$ cavity round-trip frequency with the signal-to-noise ratio of $49 \mathrm{~dB}$, which indicate the QML waveguide lasers operate at a relatively stable regime. The fundamental repetition frequency of the mode-locked laser can be calculated with the equation:

$$
f_{\text {rep }}=\frac{c}{2 n l}
$$

where $c$ is the light speed, $n$ is the waveguide refractive index, and $l$ is the length of the cavity. In this work, the laser cavity length is $9.878 \mathrm{~mm}, \mathrm{n}$ is 1.922 [43]. As a result, the fundamental repetition rate can be estimated to be as high as $\sim 7.9 \mathrm{GHz}$, which is in good agreement with the measured result. High performance of QML waveguide laser based on configuration of S-curved waveguide opens new possibilities for compact laser device.

As expected, the dual-wavelength mode-locked lasing is also observed in S-curved hexagonal optical-lattice-like waveguide No. 3 (in Figs. 9) . Fig. 9(a) depicts the measured output modelocked laser power as a function of input power through linear fitting. The lasing threshold of optical-lattice-like waveguide is $178 \mathrm{~mW}(228 \mathrm{~mW})$ and the slope efficiency is $18.65 \%$ (10.69\%) with the maximum output power of $110 \mathrm{~mW}(57 \mathrm{~mW})$ of straight (S-curved) waveguide, respectively. The dual-wavelength spectrum is shown in Fig. 9(b). Fig. 9(c) presents the pulse trains at fundamental repetition frequency of $7.94 \mathrm{GHz}$ with SNR up to $56 \mathrm{~dB}$ (Fig. 9(e)), which is in good accordance with the estimated value of $7.9 \mathrm{GHz}$. When the pump power reaches to
TABLE II

Mode-Locked Laser Performance of S-Curved WaVeguides

\begin{tabular}{|c|c|c|c|}
\hline & & $\begin{array}{c}\text { S-curved } \\
\text { Cladding } \\
\text { waveguide } \\
\end{array}$ & $\begin{array}{c}\text { S-curved } \\
\text { optical-lattice-like } \\
\text { waveguides }\end{array}$ \\
\hline \multirow{2}{*}{$\begin{array}{l}\text { Laser threshold } \\
\qquad(\mathrm{mW})\end{array}$} & Fundamental & 196 & 228 \\
\hline & $4^{\text {th }} \mathrm{HML}$ & 263 & 316 \\
\hline \multicolumn{2}{|c|}{ Maximum output power $(\mathrm{mW})$} & 77 & 57 \\
\hline \multicolumn{2}{|c|}{ Slope efficiency } & $14.1 \%$ & $10.69 \%$ \\
\hline \multirow{2}{*}{$\begin{array}{c}\text { Repetition } \\
\text { frequency }(\mathrm{GHz})\end{array}$} & Fundamental & 7.90 & 7.94 \\
\hline & $4^{\text {th }} \mathrm{HML}$ & 31.68 & 31.69 \\
\hline \multicolumn{2}{|c|}{ Signal-to-noise ratio (dB) } & 49 & 42 \\
\hline \multirow{2}{*}{ Pulse duration (ps) } & Fundamental & 64 & 64 \\
\hline & $4^{\text {th }} H M L$ & 16 & 16 \\
\hline
\end{tabular}

$316 \mathrm{~mW}$, the 4th harmonic QML is also obtained in S-curved optical-lattice-like waveguide. Fig. 9(d) illustrates the pulse trains and Fig. 9(f) depicts the generated RF spectrum of output mode-locked laser. The 4th repetition rate is measured to be $31.69 \mathrm{GHz}$ with SNR up to $42 \mathrm{~dB}$.

Table II shows the laser performance of the two types of S-curved waveguides. The optical-lattice-like waveguide shows higher laser threshold, lower slope efficiency, lower output power and SNR than those of the cladding waveguide, which can be attributed to the higher waveguide propagation loss and bending loss. The repetition frequency and pulse duration are almost same because of the same bulk sample and waveguide length.

\section{CONCLUSION}

In conclusion, we have successfully fabricated S-curved channel waveguides by direct femtosecond writing of hexagonal optical-lattice-like waveguide and depressed cladding structures. These S-curved waveguides possess low bending loss. In addition, we have realized $31.6 \mathrm{GHz}$ dual-wavelength curved waveguide laser at 1064 and $1079 \mathrm{~nm}$ with pulse duration of $16 \mathrm{ps}$, and signal-to-noise up to $49 \mathrm{~dB}$. The multi-GHz mode-locked dual-wavelength lasers could be used to generate coherent terahertz $(\mathrm{THz})$ radiations or as a laser source to detect carbon monoxide poisoning via frequency doubling. This work also indicates promising applications of S-curved waveguides for on-chip integration of ultrafast laser sources.

\section{REFERENCES}

[1] C. Grivas, "Optically pumped planar waveguide lasers: Part II: Gain media, laser systems, and applications," Prog. Quant. Electron., vol. 45-46, pp. 3-160, Jan.-Mar. 2016.

[2] C. Wieschendorf et al., "Compact integrated actively Q-switched waveguide laser," Opt. Express, vol. 25, no. 3, pp. 1692-1701, Feb. 2017.

[3] Y. Jia and F. Chen, "Compact solid-state waveguide lasers operating in the pulsed regime: A review [Invited]," Chin. Opt. Lett., vol. 17, no. 1, pp. 012302, Jan. 2019.

[4] E. Kifle et al., "Fs-laser-written erbium-doped double tungstate waveguide laser," Opt. Express, vol. 26, no. 23, pp. 30826-30836, Nov. 2018 
[5] K. van Dalfsen, S. Aravazhi, C. Grivas, S. M. García-Blanco, and M. Pollnau, "Thulium channel waveguide laser with $1.6 \mathrm{~W}$ of output power and $\sim 80 \%$ slope efficiency," Opt. Lett., vol. 39, no. 15, pp. 4380-4383, Aug. 2014

[6] S. Hakobyan, V. J. Wittwer, K. Hasse, C. Krankel, T. Sudmeyer, and T. Calmano, "Highly efficient Q-switched Yb:YAG channel waveguide laser with $5.6 \mathrm{~W}$ of average output power," Opt. Lett., vol. 41, no. 20, pp. 4715-4718, Oct. 2016.

[7] G. Salamu, F. Jipa, M. Zamfirescu, and N. Pavel, "Watt-level output power operation from diode-laser pumped circular buried depressed-cladding waveguides inscribed in Nd:YAG by direct femtosecond-laser writing," IEEE Photon. J., vol. 8, no. 1, Feb. 2016, Art. no. 1500209.

[8] T. Calmano, M. Ams, P. Dekker, M. J. Withford, and C. Kränkel, "2 W single-longitudinal-mode Yb:YAG distributed-feedback waveguide laser," Opt. Lett., vol. 42, no. 14, pp. 2734-2737, Jul. 2017.

[9] J. T. Kim, S. Park, J. J. Ju, S. Lee, and S. Kim, "Low bending loss characteristics of hybrid plasmonic waveguide for flexible optical interconnect," Opt. Express, vol. 18, no. 23, pp. 24213-24220, Nov. 2010.

[10] A. Kumar and S. Aditya, "Performance of S-bends for integrated-optic waveguides," Microw. Opt. Technol. Lett., vol. 19, no. 4, pp. 289-292, Apr. 1998.

[11] K. T. Koai and P.-L. Liu, "Modeling of Ti: $\mathrm{LiNbO}_{3}$ waveguide devices. II. S-shaped channel waveguide bends," J. Lightw. Technol., vol. 7, no. 7, pp. 1016-1022, Jul. 1989.

[12] A. Arriola et al., "Low bend loss waveguides enable compact, efficient 3D photonic chips," Opt. Express, vol. 21, no. 3, pp. 2978-2986, Feb. 2013.

[13] T. Calmano, A. G. Paschke, S. Müller, C. Kränkel, and G. Huber, "Curved Yb:YAG waveguide lasers, fabricated by femtosecond laser inscription," Opt. Express, vol. 21, no. 21, pp. 25501-25508, Oct. 2013.

[14] K. Sugioka and Y. Cheng, "Ultrafast lasers-reliable tools for advanced materials processing," Light Sci. Appl., vol. 3, Apr. 2014, Paper e149.

[15] R. R. Gattass and E. Mazur, "Femtosecond laser micromachining in transparent materials," Nat. Photon., vol. 2, pp. 219-225, Apr. 2008.

[16] D. Choudhury, J. R. Macdonald, and A. K. Kar, "Ultrafast laser inscription: Perspectives on future integrated applications," Laser Photon. Rev., vol. 8, no. 6, pp. 827-846, Jun. 2014.

[17] K. Sugioka et al., "Femtosecond laser 3D micromachining: A powerful tool for the fabrication of microfluidic, optofluidic, and electrofluidic devices based on glass," Lab Chip, vol. 14, no. 18, pp. 3447-3458, Jul. 2014.

[18] L. Li et al., "All-laser-micromachining of ridge waveguides in $\mathrm{LiNbO}_{3}$ crystal for mid-infrared band applications," Sci. Rep., vol. 7, Aug. 2017, Art. no. 7034

[19] L. Li et al., "Laser-writing of ring-shaped waveguides in BGO crystal for telecommunication band," Opt. Express, vol. 25, no. 20, pp. 24236-24241, Oct. 2017.

[20] F. Chen and J. R. Vázquez de Aldana, "Optical waveguides in crystalline dielectric materials produced by femtosecond-laser micromachining," Laser Photon. Rev., vol. 8, no. 2, pp. 251-275, May 2014.

[21] S. Gross and M. J. Withford, "Ultrafast-laser-inscribed 3D integrated photonics: Challenges and emerging applications," Nanophotonics, vol. 4, no. 3, pp. 332-352, Nov. 2015.

[22] B. Zhang et al., "Mode tailoring of laser written waveguides in $\mathrm{LiNbO}_{3}$ crystals by multi-scan of femtosecond laser pulses," Opt. Mater., vol. 86, pp. 571-575, Dec. 2018.

[23] S. Campbell et al., "Frequency-doubling in femtosecond laser inscribed periodically-poled potassium titanyl phosphate waveguides," Opt. Express, vol. 15, no. 25, pp. 17146-17150, Dec. 2007.

[24] M. Triplett et al., "Multi-watt, broadband second-harmonicgeneration in $\mathrm{MgO}$ :PPSLT waveguides fabricated with femtosecond laser micromachining," Opt. Express, vol. 27, no. 15, pp. 21102-21115, Jul. 2019.

[25] Y. Jia, C. Cheng, J. R. Vázquez de Aldana, and F. Chen, "Threedimensional waveguide splitters inscribed in Nd:YAG by femtosecond laser writing: Realization and laser emission," J. Lightw. Technol., vol. 34, no. 4, pp. 1328-1332, Feb. 2016.

[26] Y. F. Chen, "CW dual-wavelength operation of a diode-end-pumped Nd: $\mathrm{YVO}_{4}$ laser," Appl. Phys. B, vol. 70, pp. 475-478, Apr. 2000.

[27] Y. P. Huang, C. Y. Cho, Y. J. Huang, and Y. F. Chen, "Orthogonally polarized dual-wavelength $\mathrm{Nd}: \mathrm{LuVO}_{4}$ laser at $1086 \mathrm{~nm}$ and $1089 \mathrm{~nm}$," Opt. Express, vol. 20, no. 5, pp. 5644-5651, Feb. 2012.

[28] M.V. Ponarina et al., "Dual-wavelength generation of picosecond pulses with $9.8 \mathrm{GHz}$ repetition rate in Nd: YAG waveguide laser with graphene," Quantum Electron., vol. 49, no. 4, pp. 365-370, Apr. 2019.

[29] W. Q. Zhang, D. G. Lancaster, T. M. Monro, and S. A. Vahid, "Synchronised dual-wavelength mode-locking in waveguide lasers," Sci. Rep., vol. 8, pp. 7821, May 2018.
[30] D. P. Shepherd et al., "Ultrafast high-repetition-rate waveguide lasers," IEEE J. Sel. Top. Quant. Electron., vol. 43, no. 2, pp.5753-5756, Mar./Apr. 2018.

[31] S. Hakobyan et al., "Full stabilization and characterization of an optical frequency comb from a diode-pumped solid-state laser with $\mathrm{GHz}$ repetition rate," Opt. Express, vol. 25, no. 17, pp. 20437-20453, Aug. 2017.

[32] A. A. Lagatsky, A. Choudhary, P. Kannan, D. P. Shepherd, W. Sibbett, and C. T. A. Brown, "Fundamentally mode-locked, femtosecond waveguide oscillators with multi-gigahertz repetition frequencies up to $15 \mathrm{GHz}$," Opt. Express, vol. 21, no. 17, pp. 19608-19614, Aug. 2013.

[33] S. Wang et al., "Broadband few-layer $\mathrm{MoS}_{2}$ saturable absorbers," $\mathrm{Adv}$. Mater, vol. 26, pp. 3538-3544, Apr. 2014.

[34] H. Zhang et al., "Molybdenum disulfide $\left(\mathrm{MoS}_{2}\right)$ as a broadband saturable absorber for ultra-fast photonics," Opt. Express, vol. 22, no. 6, pp. 7249-7260, Mar. 2014.

[35] J. Lee, J. Koo, J. Lee, Y. M. Jhon, and J. H. Lee, “All-fiberized, femtosecond laser at $1912 \mathrm{~nm}$ using a bulk-like $\mathrm{MoSe}_{2}$ saturable absorber," Opt. Mater. Express, vol. 7, no. 8, pp. 2968-2979, Aug. 2017.

[36] R. Mary et al., "1.5 GHz picosecond pulse generation from a monolithic waveguide laser with a graphene-film saturable output coupler," Opt. Express, vol. 21, no. 7, pp. 7943-7950, Apr. 2013.

[37] F. Thorburn, A. Lancaster, S. McDaniel, G. Cook, and A. K. Kar, " 5.9 GHz graphene based q-switched mode locked mid-infrared monolithic waveguide laser," Opt. Express, vol. 25, no. 21, pp. 26166-26174, Oct. 2017.

[38] Z. Li, Y. Zhang, C. Cheng, H. Yu, and F. Chen, "6.5 GHz Q-switched modelocked waveguide lasers based on two-dimensional materials as saturable absorbers," Opt. Express, vol. 26, no. 9, pp. 11321-11330, Apr. 2018.

[39] Y. Ren et al., "7.8-GHz Graphene-based 2- $\mu \mathrm{m}$ monolithic waveguide laser," IEEE J. Sel. Top. Quantum Electron., vol. 21, no. 1, pp. 395-400, Jan./Feb. 2015.

[40] Z. Li et al., " $8.8 \mathrm{GHz}$ Q-switched mode-locked waveguide lasers modulated by $\mathrm{PtSe}_{2}$ saturable absorber," Opt. Express, vol. 27, no. 6, pp. 8727-8737, Mar. 2019.

[41] C. Grivas et al., "Generation of multi-gigahertz trains of phase-coherent femtosecond laser pulses in Ti:Sapphire waveguides," Laser Photon. Rev., vol. 12, Sep. 2018, Art. no. 1800167.

[42] W. Nie et al., "Dual-wavelength waveguide lasers at 1064 and $1079 \mathrm{~nm}$ in Nd:YAP crystal by direct femtosecond laser writing," Opt. Lett., vol. 40, no. 10, pp. 2437-2440, May 2015.

[43] Z. Zeng et al., "Measurement of the refractive index and thermal refractive index coefficients of Nd:YAP crystal," Appl. Opt., vol. 29, no. 9, pp. 1281-1286, Mar. 1990.

Lingqi Li received the B.A. degree from Shandong Normal University, Jinan, China, in 2016. She is currently working toward the Ph.D. degree under the supervision of Prof. Feng Chen with the School of Physics, Shandong University, Jinan, China. Her current research interests include femtosecond laser microand nanofabrication, waveguide lasers, and nonlinear optics.

Ziqi Li received the B.A. degree from Shandong Normal University, Jinan, China, in 2016. He is currently working toward the Ph.D. degree under the supervision of Prof. Feng Chen with the School of Physics, Shandong University, Jinan, China. His current research interests include ultrafast lasers, nonlinear optics, low dimensional materials, laser materials processing, and ion beam physics. 
Weijie Nie received the Ph.D. degree from Shandong University, Jinan, China, in 2019. She is currently a Postdoctoral Researcher with the Leibniz Institute for Solid State and Material Research (IFW), Dresden, Germany. Her current research interests include laser writing of waveguides, waveguide lasers, metallic nanoparticle, quantum dot, and entangled photon source.

Carolina Romero received the B.Sc. degree in 2006 from the University of Santiago de Compostela, Santiago, Spain, and the Ph.D. degree in 2012 from the University of Salamanca, Salamanca, Spain, where she is currently a Specialized Technician with the Laser Microprocessing Group. Her research interests include ultrafast nonlinear optics and laser microprocessing.
Javier R. Vázquez de Aldana received the B.Sc. and Ph.D. degrees from the University of Salamanca, Salamanca, Spain, in 1997 and 2001, respectively.

$\mathrm{He}$ is currently an Associate Professor with the Science Faculty, University of Salamanca. He is a member of the Laser Microprocessing Research Group and also a Technical and Scientific Advisor of the Laser Facility, University of Salamanca. His research interests include the interaction of intense femtosecond pulses with materials and its application to fabrication of photonic devices.

Feng Chen (Senior Member, OSA) received the Ph.D. degree from Shandong University, Jinan, China, in 2002.

$\mathrm{He}$ is currently a Professor with the School of Physics, Shandong University. From 2003 to 2005, he was with the Clausthal University of Technology, Germany, as an Alexander von Humboldt Research Fellow. His research interests include material modifications by ultrafast lasers and ion beams, optical waveguides, 2D materials, plasmonics, nonlinear optics, and lasers. He is a Fellow of the Institute of Physics (IOP), U.K., a Senior Member of the Optical Society of America (OSA), SPIE, and a Member of the Board of Directors, Chinese Physical Society. He is the Deputy Editor of Chinese Optics Letters, and also serves as an Editorial Board Member of Optical Engineering and Scientific Reports. 